University of Wollongong

Research Online

Faculty of Engineering and Information

Faculty of Engineering and Information

Sciences - Papers: Part A

Sciences

$1-1-2014$

Coherent and tunable terahertz radiation from graphene surface plasmon polaritons excited by an electron beam

\author{
Shenggang Liu \\ University of Electronic Science and Technology of China \\ Chao Zhang \\ University of Wollongong, czhang@uow.edu.au \\ Min $\mathrm{Hu}$ \\ University of Electronic Science and Technology of China \\ Xiaoxing Chen \\ University of Electronic Science and Technology of China \\ Ping Zhang \\ University of Electronic Science and Technology of China, pz939@uow.edu.au
}

See next page for additional authors

Follow this and additional works at: https://ro.uow.edu.au/eispapers

Part of the Engineering Commons, and the Science and Technology Studies Commons

Research Online is the open access institutional repository for the University of Wollongong. For further information contact the UOW Library: research-pubs@uow.edu.au 


\title{
Coherent and tunable terahertz radiation from graphene surface plasmon polaritons excited by an electron beam
}

\author{
Abstract \\ Although surface plasmon polaritons (SPPs) resonance in graphene can be tuned in the terahertz regime, \\ transforming such SPPs into coherent terahertz radiation has not been achieved. Here, we propose a \\ graphene-based coherent terahertz radiation source with greatly enhanced intensity. The radiation works \\ at room temperature, it is tunable and can cover the whole terahertz regime. The radiation intensity \\ generated with this method is 400 times stronger than that from SPPs at a conventional dielectric or \\ semiconducting surface and is comparable to that from the most advanced photonics source such as a \\ quantum cascade laser. The physical mechanism for this strong radiation is presented. The phase \\ diagrams defining the parameters range for the occurrence of radiation is also shown.
}

\section{Keywords}

coherent, graphene, electron, surface, tunable, terahertz, radiation, plasmon, polaritons, excited, beam

\section{Disciplines}

Engineering | Science and Technology Studies

\section{Publication Details}

Liu, S., Zhang, C., Hu, M., Chen, X., Zhang, P., Gong, S., Zhao, T. \& Zhong, R. (2014). Coherent and tunable terahertz radiation from graphene surface plasmon polaritons excited by an electron beam. Applied Physics Letters, 104 (20), 201104-1-201104-5.

\section{Authors}

Shenggang Liu, Chao Zhang, Min Hu, Xiaoxing Chen, Ping Zhang, Sen Gong, Tao Zhao, and Renbin Zhong 


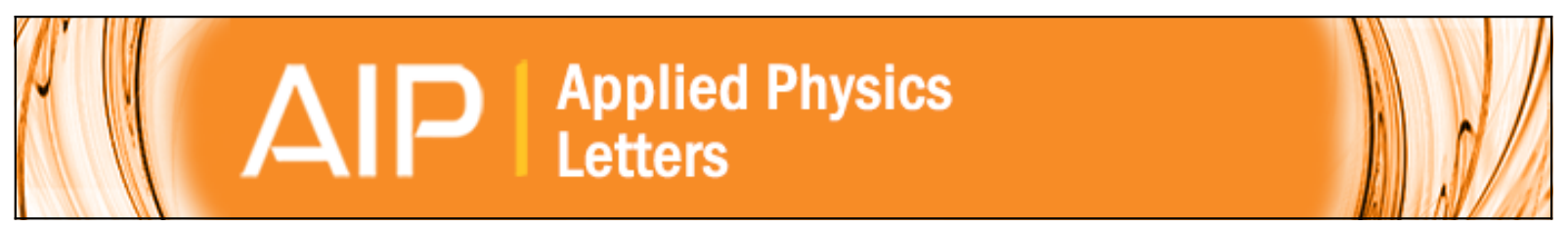

Coherent and tunable terahertz radiation from graphene surface plasmon polaritons excited by an electron beam

Shenggang Liu, Chao Zhang, Min Hu, Xiaoxing Chen, Ping Zhang, Sen Gong, Tao Zhao, and Renbin Zhong

Citation: Applied Physics Letters 104, 201104 (2014); doi: 10.1063/1.4879017

View online: http://dx.doi.org/10.1063/1.4879017

View Table of Contents: http://scitation.aip.org/content/aip/journal/apl/104/20?ver=pdfcov

Published by the AIP Publishing

\section{Articles you may be interested in}

Highly efficient, broadband coherent surface-mixing-wave generation using amplified surface plasmonic polaritons

Appl. Phys. Lett. 104, 203108 (2014); 10.1063/1.4878406

Surface plasmon polaritons on soft-boundary graphene nanoribbons and their application in switching/demultiplexing

Appl. Phys. Lett. 103, 133104 (2013); 10.1063/1.4822044

Extremely confined terahertz surface plasmon-polaritons in graphene-metal structures

Appl. Phys. Lett. 103, 071103 (2013); 10.1063/1.4818660

Terahertz plasmonics in ferroelectric-gated graphene

Appl. Phys. Lett. 102, 201118 (2013); 10.1063/1.4807762

Efficient manipulation of surface plasmon polariton waves in graphene

Appl. Phys. Lett. 100, 243110 (2012); 10.1063/1.4729557

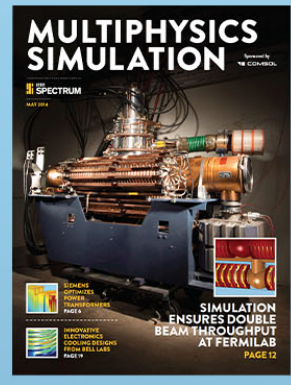

Free online magazine

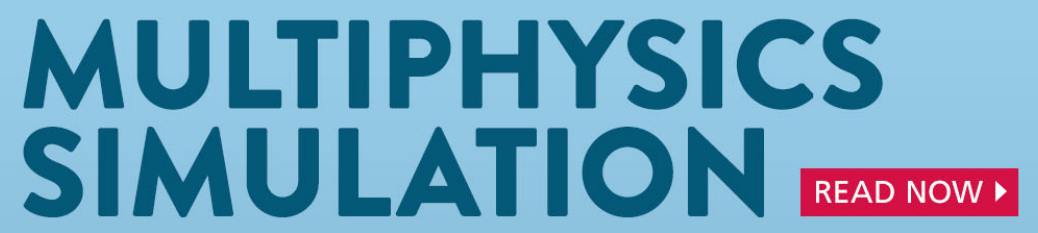




\title{
Coherent and tunable terahertz radiation from graphene surface plasmon polaritons excited by an electron beam
}

\author{
Shenggang Liu, ${ }^{1,2, a)}$ Chao Zhang, ${ }^{1,2,3}$ Min Hu, ${ }^{1,2}$ Xiaoxing Chen, ${ }^{1,2}$ Ping Zhang, ${ }^{1,2}$ \\ Sen Gong, ${ }^{1,2}$ Tao Zhao, ${ }^{1,2}$ and Renbin Zhong ${ }^{1,2}$ \\ ${ }^{1}$ Terahertz Research Centre, School of Physical Electronics, University of Electronic Science and Technology \\ of China, Chengdu, Sichuan 610054, China \\ ${ }^{2}$ Cooperative Innovation Centre of Terahertz Science, Chengdu, Sichuan 610054, China \\ ${ }^{3}$ School of Physics and Institute for Superconducting and Electronic Materials, University of Wollongong, \\ New South Wales 2522, Australia
}

(Received 31 March 2014; accepted 7 May 2014; published online 20 May 2014)

\begin{abstract}
Although surface plasmon polaritons (SPPs) resonance in graphene can be tuned in the terahertz regime, transforming such SPPs into coherent terahertz radiation has not been achieved. Here, we propose a graphene-based coherent terahertz radiation source with greatly enhanced intensity. The radiation works at room temperature, it is tunable and can cover the whole terahertz regime. The radiation intensity generated with this method is 400 times stronger than that from SPPs at a conventional dielectric or semiconducting surface and is comparable to that from the most advanced photonics source such as a quantum cascade laser. The physical mechanism for this strong radiation is presented. The phase diagrams defining the parameters range for the occurrence of radiation is also shown. (C) 2014 AIP Publishing LLC. [http://dx.doi.org/10.1063/1.4879017]
\end{abstract}

Graphene has become the most attractive research area in modern science and technology because of its exceptional properties $^{1-3}$ and great potential applications. ${ }^{4-7}$ One of the most interesting and important topics in graphene applications is to generate terahertz $(\mathrm{THz})$ radiation, for that the plasmon frequency of graphene with favourable charge carrier concentration lies in the $1-50 \mathrm{THz}$ frequency regime. ${ }^{8}$ Graphene-based plasmonics can play a versatile role for developing photonic and optoelectronic applications, such as ultrafast lasers, solar cells, optical modulators, photodetectors, and light emitting devices. ${ }^{9}$ It has been proven both theoretically and experimentally that graphene can sustain surface plasmon polaritons (SPPs) while the real part of the permittivity becomes negative. ${ }^{3,7}$

From the SPPs behavior and the excitation methods, graphene sheet is similar to a thin noble metal film. For example, graphene SPPs can also be excited by incident plane wave, ${ }^{10,11}$ strong near-field, ${ }^{12}$ and electron beam. ${ }^{13}$ It also has been shown that, in a noble metal film, SPPs can be used to generate light radiation. ${ }^{14}$ Moreover, graphene SPPs have many advantages over metal film SPPs because the conductivity and permittivity of graphene can be controlled by adjusting external gate voltage, chemical doping, etc. ${ }^{3,7}$ Various approaches have been proposed and investigated to generate $\mathrm{THz}$ radiation. ${ }^{8,15,16}$ Despite the recent intensive research, generation of coherent and tunable $\mathrm{THz}$ radiation using graphene remains a significant challenge.

In this Letter, we present a physical mechanism for generation of tunable and coherent THz radiation from SPPs in graphene. The mechanism consists of three elements: (i) Exciting SPPs in graphene by a uniformly moving electron beam and the SPPs propagate with the electron beam, (ii) the periodicity of the dielectrics to bring the SPP-beam line

a)liusg@uestc.edu.cn intersection at short wavelength back to the long wavelength regime for the SPPs to be transformed into radiation waves, and (iii) the strong electron-photon and SPP-photon coupling in graphene.

Let us first consider a structure of graphene sheet on a dielectric substrate (such as $\mathrm{Si}, \mathrm{SiO}_{2}$, and Polymethylpentene $(\mathrm{TPX})^{17}$ ) as shown in Fig. 1(a). SPPs in graphene are being excited by an electron beam that moves parallel above the graphene. The conductivity of graphene $\left(\sigma_{\mathrm{g}} \approx \sigma_{\text {intra }}\right)$ is given as $3,18,19$

$$
\sigma_{\mathrm{g}}=\frac{j e^{2} k_{B} T}{\pi \hbar^{2}\left(\omega+j \tau^{-1}\right)}\left[\frac{\mu_{c}}{k_{B} T}+2 \ln \left(e^{-\frac{\mu_{c}}{k_{B} T}}+1\right)\right]
$$

where $T$ is temperature, $k_{B}$ is Boltzmann constant, $\tau$ is relaxation time, and $\mu_{c}$ is chemical potential. Values of $\mu_{c}=(0.08-0.15) \mathrm{eV}, \tau=0.5 \mathrm{ps}$, and $T=300 \mathrm{~K}$ are used for all numerical calculations. ${ }^{18}$ Here, only the intraband conductivity which dominates the low frequency process of graphene SPP is included. ${ }^{19}$ When graphene dynamical intraband conductivity has a positive imaginary part, the real part of the equivalent permittivity becomes negative. In this case, graphene can support SPPs. In deriving the dispersion equation of graphene SPPs of the structure excited by an electron beam, as shown in Fig. 1(a), the graphene sheet is considered as a conducting surface with conductivity $\sigma_{g}$. By making use of Maxwell's equations and the boundary conditions, the dispersion equation of SPPs in graphene with dielectric substrate can be obtained as ${ }^{20}$

$$
\frac{1}{\sqrt{k_{0}^{2}-k_{z}^{2}}}+\frac{\varepsilon_{s}}{\sqrt{\varepsilon_{s} k_{0}^{2}-k_{z}^{2}}}+\frac{\sigma_{g}}{\omega \varepsilon_{0}}=0
$$

where $\varepsilon_{s}$ is the relative dielectric constant of substrate.

The dispersion curves of the structure with different chemical potentials are plotted shown in Fig. 1(b). The 
electron beam with $\beta=0.04$ (where $\beta=u_{0} / c, u_{0}$ is the beam velocity) is moving parallel above the graphene sheet. As shown in Fig. 1(b), the beam line intersects dispersion curves at points A and B which are called "working points." It indicates that graphene SPPs are excited at frequency $8.44 \mathrm{THz}$ for $\mu_{c}=0.15 \mathrm{eV}$ and $5.68 \mathrm{THz}$ for $\mu_{c}=0.1 \mathrm{eV}$ corresponding to working points $\mathrm{A}$ and $\mathrm{B}$, respectively. The frequency of graphene SPPs can be tuned by the beam energy or the chemical potential (gate voltage) to cover almost the whole $\mathrm{THz}$ regime.

In the case of parallel electron beam excitation, graphene SPPs can propagate together with the electron beam for that their phase velocity equals the velocity of electron beam. The SPPs can obtain energy continuously from electron beam to compensate their energy loss during propagation. Fig. 1(c) clearly shows the graphene SPPs propagation and SPPs are tightly confined to the both sides of graphene sheet.

The SPPs cannot radiate directly from the graphene sheet. It could be transformed into Cherenkov radiation, if the working points are in the radiation region, a cone formed by the vacuum light line. ${ }^{14}$ However, the SPPs dispersion curves shown in Fig. 1(b) are always below the dielectric light line, so the intersections of SPPs dispersion curves and the beam line are always below the radiation region, irrespective of whether the permittivity of the dielectric substrate is lower or higher. Therefore, it is not possible to transform graphene SPPs into radiation because of the wavelength mismatch.

We now propose a structure in which the wavelength mismatch can be removed and the SPP-beam line intersection can be brought back within the cone region formed by the light lines. The schematic of this structure is shown in Fig. 2(a). Here, a micrometer slits array is used as the substrate, it is a periodic structure formed by dielectric medium 1 and medium 2 alternatively. The height of dielectric slits array is set to be comparable to the length of period to ensure high radiation power density and independence of radiation frequency on substrate below dielectric slits array. ${ }^{20}$ From Maxwell's equations together with the boundary conditions, we obtain the dispersion equation ${ }^{20}$

$$
\frac{\varepsilon_{e f f} k_{3}+\varepsilon_{s} k_{2}}{\varepsilon_{e f f} k_{3}-\varepsilon_{s} k_{2}} e^{-2 j k_{2} d}=\frac{\varepsilon_{e f f} k_{0} k_{1}-\eta_{0} \sigma_{g} k_{1} k_{2}-k_{0} k_{2}}{\varepsilon_{e f f} k_{0} k_{1}+\eta_{0} \sigma_{g} k_{1} k_{2}+k_{0} k_{2}},
$$

where $k_{1}=\sqrt{k_{0}^{2}-k_{z}^{2}}, k_{2}=\sqrt{\varepsilon_{e f f} k_{0}^{2}-k_{z}^{2}}, k_{3}=\sqrt{\varepsilon_{s} k_{0}^{2}-k_{z}^{2}}$, $\varepsilon_{e f f}=\varepsilon_{s}(1-r)+\varepsilon_{2} r, r=a_{2} / L, \eta_{0}$ is the wave impendence in a vacuum, and $\varepsilon_{e f f}$ is the effective dielectric function of the dielectric array substrate. The dispersion curves are shown in Fig. 2(b). In analog to the translational invariance of a crystal lattice, the periodicity of the dielectric media results in zone folding of the SPPs dispersion. The zone boundary is given by $k_{L}=\pi / L$, and the zone folding $\left(k_{n}=k \pm 2 n \pi / L\right)$ occurs. Since the speed of light is much higher than the electron speed, the cone formed by the light lines is essentially a very narrow region near the zone center. Therefore, it is required that the SPP-beam line intersection point be very close to the second zone boundary $\left(2 k_{L}\right)$, so it can be folded back to the zone center (inside the light cone). As a result, transforming SPPs to radiation becomes possible. Fig. 2(b) shows that in this case the intersections of the dispersion curves and the (a)
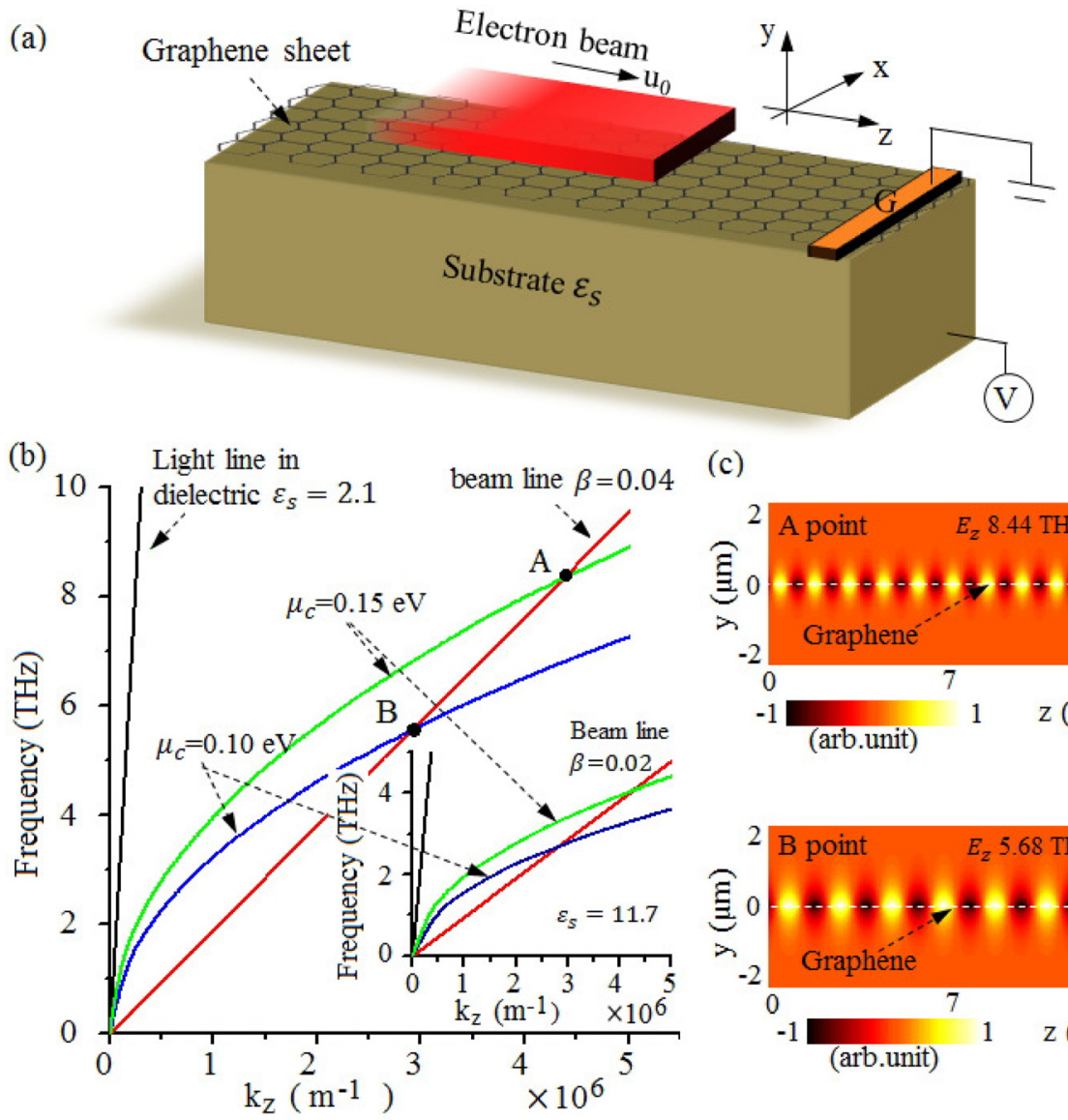

(c)
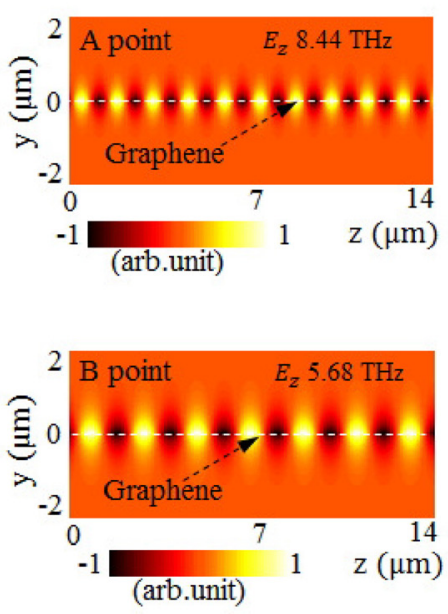

FIG. 1. (a) A monolayer graphene is on a dielectric substrate and an electron beam uniformly moves parallel above the graphene. (b) The dispersion curves of the structure with the substrate $\varepsilon_{s}=2.1$ (TPX). The light line in vacuum almost coincides with that in dielectric. The inset is the dispersion curve for the substrate $\varepsilon_{s}=11.7(\mathrm{Si})$. (c) The contour map of graphene SPPs at working points $\mathrm{A}$ and $\mathrm{B}$. 
(a)

(a) Graphene sheet

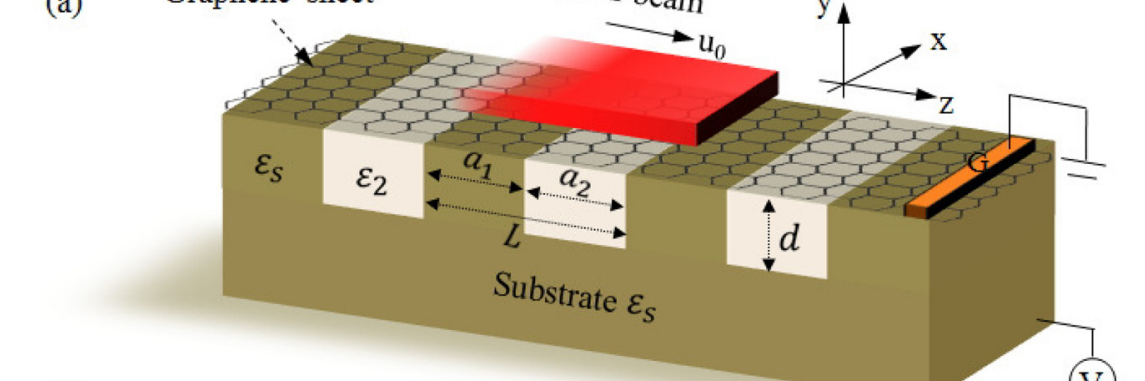

(b)

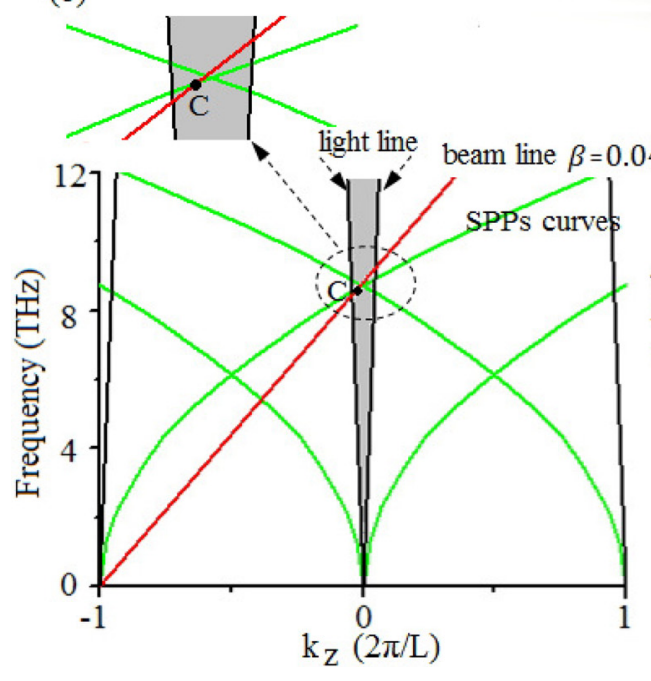

(c)

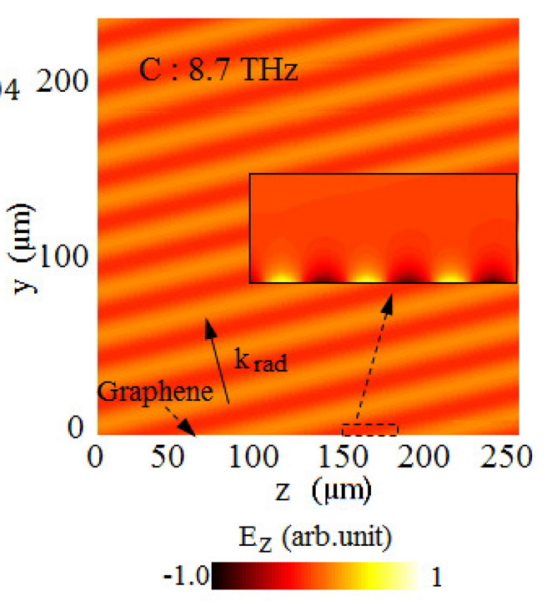

FIG. 2. (a) The schematic of graphene sheet with a micrometer dielectric slits array substrate. (b) The dispersion curves (the geometric size is $L=1.5$ $\mu \mathrm{m}, d=1 \mu \mathrm{m}, a_{1}=1 \mu \mathrm{m}$, and $a_{2}=0.5$ $\mu \mathrm{m}, \varepsilon_{s}=2.1, \varepsilon_{2}=1, \beta=0.044$ ). (c) The contour map of $E_{z}$ field at working point $\mathrm{C}(8.7 \mathrm{THz}), k_{\text {rad }}=k_{0}$ is the radiation wave vector in the free space, and the inset is enlarged view near the graphene sheet. beam line can be in the radiation region between the two vacuum light lines, and hence, SPPs can be transformed into coherent radiation in free space, the radiation pattern is shown in Fig. 2(c). The radiation frequency is in the THz frequency regime, and it is tunable by changing the beam energy. A tunable range of $\triangle f / f_{0}=5 \%$ can be achieved.

The dependence of the radiation frequency on various different parameters is shown in Fig. 3. Fig. 3(a) shows that as the chemical potential increases, the surface plasmon frequency (proportional to the square-root of the chemical potential) as well as the SPPs energy increase. This leads to a higher intersection point with the electron beam line, and in turn a higher radiation frequency. To get better operation, the electron beam energy needs to be adjusted within an operational band for a given chemical potential. The radiation condition is determined by the intersection of SPP energy and the beam line and the intersection point must be folded back to a position inside the cone formed by the light lines. If the SPP energy increases (as the chemical potential increases), the beam energy must increase to insure that the intersection point is close to the second zone boundary. Then the folding will bring the intersection point inside the light cone. The colored 3D band shows the required SPP and beam energy matching. A cut at a fixed radiation frequency will give a $\beta-\mu_{c}$ phase diagram for radiation.

Figs. 3(b) and 3(c) show the effect of the device geometry on the radiation frequency. The zone folding due to the periodic structure of the dielectrics is characterized by the wave-vector at the second zone boundary, $2 k_{L}$. The SPPs energy at $2 k_{L}$ therefore increases as the period $L$ decreases. A large period results a low intersection point when the dispersion folds back to the zone center. In general, the radiation frequency decreases with the increasing period. In the case of $\varepsilon_{S}>\varepsilon_{2}$, the frequency increases as ratio $a_{2} / L$ increases because of the decrease of the effective dielectric constant $\varepsilon_{e f f}$. It is found that the radiation frequency can be tuned to as low as $3 \mathrm{THz}$. Tuning radiation frequency with periodicity is also constrained by the beam energy. If $k_{L}$ is increased, $\beta$ needs to increase accordingly so the intersection point stays close to the second zone boundary. A cut at fixed frequency
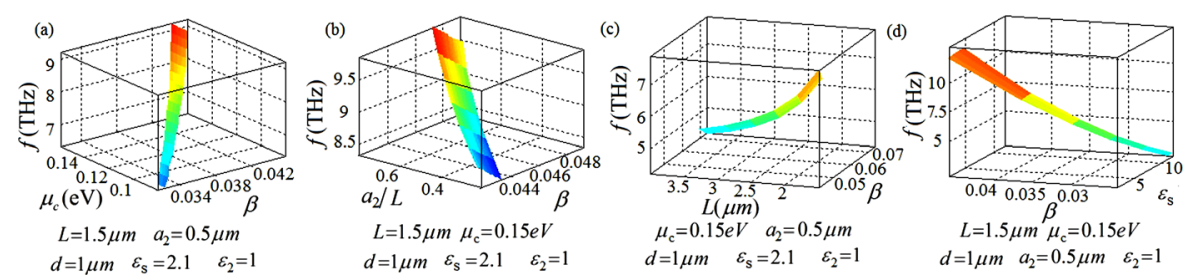

FIG. 3. The dependence of the radiation frequency on various different parameters. (a) Radiation frequency vs. chemical potential and beam energy; (b) radiation frequency vs. ratio of slit width to period and beam energy; (c) radiation frequency vs. period and beam energy; (d) radiation frequency vs. relative permittivity and beam energy. 
in the 3D plot of Figs. 3(b) and 3(c) represents a $k_{L}-\beta$ phase diagram defining radiation occurrence. The dependence of the radiation frequency on the relative permittivity is shown in Fig. 3(d). The radiation frequency decreasing with the relative permittivity is due to the surface plasmon energy decreasing with the relative permittivity. When all other parameters are fixed, $\beta$ has the largest tuning range against the chemical potential. The dependence of radiation power density on the chemical potential and geometric parameters of the periodic slits array can be found in Ref. 20. Therefore, adjusting the parameters of this structure, the radiation frequency may cover almost the whole $\mathrm{THz}$ regime.

To ensure completeness of our discussion, we have also evaluated the performance of graphene SPP when the dielectric slits array is replaced by a conducting micrometer slits array. Our result shows that no better performance can be obtained.

The output power density of this radiation can be calculated by the following integral over a unit cell: ${ }^{20}$

$$
P_{d}=-\frac{1}{2} \operatorname{Re}\left[\iint \sum_{n} \frac{\omega \varepsilon_{0} A_{1 n}^{2}}{k_{1 n}} d S\right],
$$

where $A_{1 n}$ is the coefficient of the radiation field. The numerical calculations show that the power density for a monolayer graphene with dielectric slits array substrate can reach $100 \mathrm{~mW} / \mathrm{cm}^{2}$ with electron charge density of $10^{-2} \mathrm{pC} / \mathrm{cm}$; it is comparable with other photonic THz radiation sources. ${ }^{21}$ In our case, the size of the $\mathrm{THz}$ radiation structure is miniature, micrometer size, and it can be easily integrated to get higher radiation power.

Fig. 4 shows the radiation field for the dielectric slits array structure with and without graphene. It shows that up to 20 times high amplitude enhancement of the radiation can be achieved. The 20 times amplitude enhancement revealed here indicates that the strength of the light-matter interaction in graphene is 20 times stronger than that in most conventional semiconductors. We note that the optical response of a single layer graphene is about 10 to 100 times stronger than that in conventional semiconductors, as shown by the exceptional strong absorption rate of $2.3 \%$ per atomic layer.

The physics of the enhancement is investigated to reveal what substantial role SPPs in the graphene sheet play to

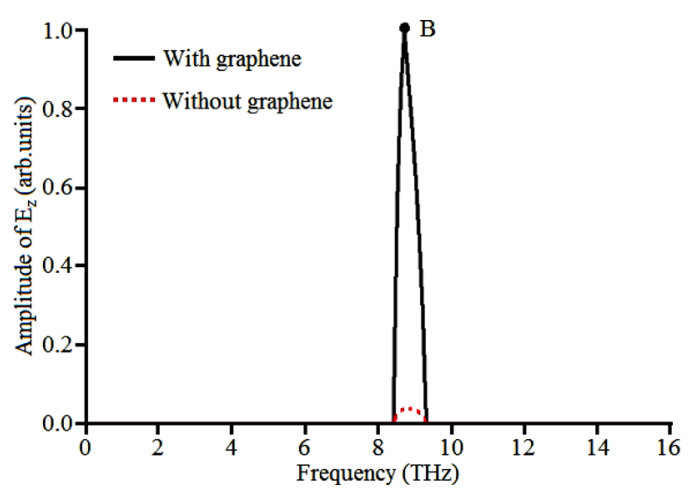

FIG. 4. The spectrum of the radiation field $\left(E_{z}\right)$ in the upper space (vacuum) for the dielectric slits array with graphene (black line) and without graphene (red dotted line). The calculated result is based on Eq. (II.9) in Appendix II of Ref. 20, and the parameters are the same as those used in Fig. 2. enhance the radiation. Graphene SPPs can be considered as an equivalent surface current source: ${ }^{10} J_{z s p}=\sigma_{g} E_{z}$, where $\sigma_{g}$ is the conductivity of graphene and $E_{z}$ is electron beam generated field penetrated into the graphene. Theory developed here shows that the radiation field contributed by the equivalent current density $J_{z s p}$ is much higher than that by the electron beam at the working frequency. As a result, the total radiation power is dramatically enhanced. Based on this calculation, up to 400 times enhancement of the radiation power density can be achieved. ${ }^{20}$

In summary, we revealed a physical scheme of coupling evanescent wave from parallel moving electron beam into graphene SPPs, and we found that graphene SPPs in a structure with periodic dielectric slits can be transformed into tunable coherent $\mathrm{THz}$ radiation. The radiation characteristics can be tuned electrically by adjusting beam energy or chemical potential, and the radiation frequency covers the whole $\mathrm{THz}$ regime. The most significant result is that radiation intensity is enhanced by 400 times by SPPs confined to graphene. Based on this radiation mechanism, a room temperature, tunable, coherent, and miniature $\mathrm{THz}$ radiation source can be developed.

This work was supported by the National Key Programme of Fundamental Research of China under Contract Nos. 2014CB339801 and 2007CB310401, the Natural Science Foundation of China under Grant Nos. 61231005, 11305030, and 612111076, and National High-tech Research and Development Project under Contract No. 2011AA010204. Acknowledgement should also be extended to Professor Xingbi Chen for his valuable discussions and suggestions.

${ }^{1}$ A. C. Neto, F. Guinea, N. Peres, K. S. Novoselov, and A. K. Geim, Rev. Mod. Phys. 81, 109 (2009).

${ }^{2}$ K. Novoselov, A. K. Geim, S. Morozov, D. Jiang, M. Katsnelson, I. Grigorieva, S. Dubonos, and A. Firsov, Nature 438, 197 (2005).

${ }^{3}$ J. Horng, C.-F. Chen, B. Geng, C. Girit, Y. Zhang, Z. Hao, H. A. Bechtel, M. Martin, A. Zettl, M. F. Crommie et al., Phys. Rev. B 83, 165113 (2011).

${ }^{4}$ M. Liu, X. Yin, E. Ulin-Avila, B. Geng, T. Zentgraf, L. Ju, F. Wang, and X. Zhang, Nature 474, 64 (2011).

${ }^{5}$ L. Ju, B. Geng, J. Horng, C. Girit, M. Martin, Z. Hao, H. A. Bechtel, X. Liang, A. Zettl, Y. R. Shen et al., Nat. Nanotechnol. 6, 630 (2011).

${ }^{6}$ K. Yang, S. Arezoomandan, and B. Sensale-Rodriguez, Terahertz Sci. Technol. 6, 223 (2013).

${ }^{7}$ A. Vakil and N. Engheta, Science 332, 1291 (2011).

${ }^{8}$ M. Ryzhii and V. Ryzhii, Jpn. J. Appl. Phys., Part 2 46, L151 (2007).

${ }^{9}$ A. Grigorenko, M. Polini, and K. Novoselov, Nat. Photonics 6, 749 (2012).

${ }^{10}$ W. Gao, G. Shi, Z. Jin, J. Shu, Q. Zhang, R. Vajtai, P. M. Ajayan, J. Kono, and Q. Xu, Nano Lett. 13, 3698 (2013).

${ }^{11}$ Y. V. Bludov, A. Ferreira, N. Peres, and M. Vasilevskiy, Int. J. Mod. Phys. B 27, 1341001 (2013).

${ }^{12}$ Z. Fei, A. S. Rodin, G. O. Andreev, W. Bao, A. S. McLeod, M. Wagner, L. M. Zhang, Z. Zhao, M. Thiemens, G. Dominguez, et al., Nature 487, 82 (2012).

${ }^{13}$ W. Zhou, J. Lee, J. Nanda, S. T. Pantelides, S. J. Pennycook, and J.-C. Idrobo, Nat. Nanotechnol. 7, 161 (2012).

${ }^{14}$ S. Liu, P. Zhang, W. Liu, S. Gong, R. Zhong, Y. Zhang, and M. Hu, Phys. Rev. Lett. 109, 153902 (2012).

${ }^{15}$ T. Watanabe, T. Fukushima, Y. Yabe, S. A. B. Tombet, A. Satou, A. A. Dubinov, V. Y. Aleshkin, V. Mitin, V. Ryzhii, and T. Otsuji, New J. Phys. 15, 075003 (2013).

${ }^{16}$ K. Tantiwanichapan, J. DiMaria, S. N. Melo, and R. Paiella, Nanotechnology 24, 375205 (2013).

${ }^{17}$ J. W. Suk, A. Kitt, C. W. Magnuson, Y. Hao, S. Ahmed, J. An, A. K. Swan, B. B. Goldberg, and R. S. Ruoff, ACS Nano 5, 6916 (2011). 
${ }^{18}$ B. Wang, X. Zhang, X. Yuan, and J. Teng, Appl. Phys. Lett. 100, 131111 (2012).

${ }^{19}$ G. W. Hanson, J. Appl. Phys. 103, 064302 (2008).

${ }^{20}$ See supplementary material at http://dx.doi.org/10.1063/1.4879017 for: (I) Graphene with dielectric substrate; (II) graphene with dielectric micrometer slits array; (III), graphene with PEC micrometer slits array; and (IV) the mechanism of enhancement.

${ }^{21}$ M. Brandstetter, C. Deutsch, M. Krall, H. Detz, D. C. MacFarland, T. Zederbauer, A. M. Andrews, W. Schrenk, G. Strasser, and K. Unterrainer, Appl. Phys. Lett. 103, 171113 (2013). 